University of South Florida

DIGITAL COMMONS

Digital Commons @ University of

@ UNIVERSITY OF SOUTH FLORIDA

South Florida

QMaSC: A Handbook for Directors of

Quantitative and Mathematics Support Centers

USF Libraries

$1-1-2016$

\title{
21. Designing a QMaSC Space
}

Eileen M. Phillips

Keene State College

Follow this and additional works at: https://digitalcommons.usf.edu/qmasc_handbook

\section{Recommended Citation}

Eileen M. Phillips (2016), "Designing a QMaSC Space", http://dx.doi.org/10.5038/9780977674435.ch21 in G. Coulombe, M. O'Neill, M. Schuckers (Eds.) A Handbook for Directors of Quantitative and Mathematical Support Centers, Neck Quill Press, http://scholarcommons.usf.edu/qmasc_handbook.

This Starting a Center is brought to you for free and open access by the USF Libraries at Digital Commons @ University of South Florida. It has been accepted for inclusion in QMaSC: A Handbook for Directors of Quantitative and Mathematics Support Centers by an authorized administrator of Digital Commons @ University of South Florida. For more information, please contact digitalcommons@usf.edu. 


\section{Designing a QMaSC Space}

(c) Eileen M. Phillips,

Keene State College

吅

\section{Introduction}

The design, location, and layout of a quantitative or mathematics support center (QMaSC) are key factors to a successful center. A positive experience at the center will encourage students to return and continue to seek help to understand the math and quantitative reasoning in their courses. "It is not just a space that is being created, but an environment that encourages and supports learning of a subject that leaves many wanting for understanding" [1].

In the design of a space for a quantitative or mathematics support center, many questions arise. Should the center stand alone or be with other support services in a large learning center? How large a space is needed for the center? Where on the campus should the center be located? What type of furniture is needed? How can the space attract students to use the center? All centers are different and reflect the culture and needs of each campus.

Much research has been conducted on the effectiveness of mathematics tutoring, but very little research has focused on the best practices in designing a tutoring center. Therefore, studies of space planning for effective learning spaces at institutions were consulted and adapted for use in this chapter. Most spaces are designed by those who will supervise the space rather than those who will use the space. If at all possible, a director should involve the potential users of the QMaSC early in the process to help inform decisions [2].

Suggested Citation: Eileen M. Phillips (2016), "Designing a QMaSC Space", http://dx.doi.org/10.5038/ 9780977674435.ch21 in G. Coulombe, M. O'Neill, M. Schuckers (Eds.) A Handbook for Directors of Quantitative and Mathematical Support Centers, Neck Quill Press, http://scholarcommons.usf.edu/qmasc_handbook.

This material is based upon work supported, in part, by the National Science Foundation under Grant DUE1255945. Any opinions, findings, and conclusions or recommendations expressed in this material are those of the author(s) and do not necessarily reflect the views of the National Science Foundation 
Two principal components will be addressed within this chapter: space planning and designing a center. The first component, space planning, involves those aspects of the center that relate directly to the physical space on campus that will be used for the center. The second component, designing a center, involves those arrangements needed to ensure a comfortable and fully functioning center environment.

\section{Space Planning for a QMaSC}

When designing a QMaSC space, a committee, including the director, the academic dean, representatives from the physical plant, and other stakeholders should start by looking at the overall culture of the campus. Every institution will create its space in a unique way based on its strengths and weaknesses as well as the resources and infrastructure available [3]. To help create that space, visit centers at other institutions and then create a list of pros and cons to determine what features would work best given the space and resources of the new center.

An important step in planning for a center is to survey potential users to determine what spaces they find most beneficial to learning. This may not always be possible, particularly if there isn't enough time or money to complete and analyze a survey. A 2008 survey completed by the Society for College and University Planning (SCUP) and Herman Miller, Inc. can be used to identify the most important characteristics for learning spaces on a campus. These characteristics emerged: spaces need to be flexible, to promote social interaction and healthful living, to use available assets and be sustainable, and to be attractive and stimulate creative thinking [2]. Special consideration must be given to individuals with special needs. A helpful resource that contains information about the legal issues involved in creating a space that is accessible to everyone as well as a checklist that can be used when designing a space can be found at: http://www.washington.edu/doit/programs/accesscollege/ student-services-conference-room/accommodations/tutoring-and-learning-centers [4].

The location of the center is another important consideration in the planning phase. Each director will need to think about the culture of the campus to determine which location is best. Is the QMaSC in a central location, such as a library or a learning center with a coordinator and all support services involved, or is it located in the mathematics department [1, 5]? Housing the center within the mathematics department puts tutors close to faculty members who can assist in answering questions and shows the commitment of the department to the quantitative needs of the campus. However, centers within departments may not be open for extensive hours, and the mathematics department may not be located in a central space on campus. Some institutions may find it more attractive to have the center in a centralized location such as the library, that is open many more hours of the week and is in the center of the campus where it is easily accessible to students. Students may also be more willing to visit a center where they won't run into their mathematics 
professor [3]. The downside to housing the center away from the mathematics department is that mathematics faculty members may not have time to visit frequently or to get to know how the center is functioning.

Visibility of a QMaSC on campus is critical. Support centers tucked away are hard to find, and they provide an excuse for students not to visit. Students who are having difficulty understanding the quantitative components of a class often feel anxious about their lack of understanding and feel awkward about getting help. According to Halcrow and Iiams, students felt a sense of intimidation asking for help from peers who excelled in mathematics, and they were also reluctant to visit an unfamiliar place with unknown people. They found students were "more willing to risk their success in the course than to risk being perceived at a lower status in mathematics than their peers" [6]. They also found that lower-ability students were the most hesitant to seek help, even though they were the population who needed it most. Centers must be very visible on campus so that when students find the courage to visit the center, they can do so quickly and easily.

The size and functions of the center should also be considered. Will more than just tutoring take place in the center? Will there be testing, study sessions, group work, meetings, and classes also be using the space? How will all of these functions work in the allotted space? Many centers will be located in existing spaces and will have to manage with what is given, but if new construction is a possibility, try to allow for the most versatile spaces. Versatility will allow for the space to get the most use possible as well as allow growth of the center through programmatic changes. The director of the center will need to produce a schedule that allows all of the functions of the center to coexist harmoniously.

The director or designer should decide at the onset whether or not there will be separate spaces for mathematics majors to work and for non-majors to be tutored. If there is only one room for tutoring, there is the risk that math majors will visit their friends who are on tutoring duty to work together on assignments. Although such visits might help build a community of learners, it is a problem if students who seek help at the center are overwhelmed by the level of mathematics that is being discussed or distracted by the noise created during the process of working on higher level mathematics problems. Apprehensive students do not want to enter such an environment. If two rooms are not established at the beginning, students may become comfortable in one space and not want to leave. It is best to start out with two separate rooms and make the purposes of the spaces known from the outset. Croft [3] cites a center that dedicated one room to be used for tutoring only, and an adjoining room to be used for individual or small group work, rather than trying to repurpose a space later for majors to complete their work. Solomon [1] found that when second and third year math majors were asked to use a new space to make more room for the first-year math majors and non-majors in the tutoring center, it disrupted the "strong collaborative working system" that had developed. This change caused a polarizing effect in that some students worked in the new room provided, while others stopped visiting the center altogether. Hunley and 
Schaller [7] also found that students are less likely to use a space for a new purpose if it has not been physically changed in some manner. They also found that students wanted permission to use the space in a different way before they felt comfortable changing the established rules of a prior space.

\section{Designing Center Space}

Hunley and Schaller [7] used multiple methods to collect data to discover what the best spaces were for encouraging student engagement. Two of their findings were related to students and their workspaces: (1) students reacted best to a tutoring experience that was serious, to tutors who treated them with respect and encouraged interaction with other students, and (2) students were more engaged in the learning process if the physical characteristics of the space were welcoming. Carroll and Gill [8] also found in their research that a center needed to create an environment "where students feel no question is a stupid question" and that students valued the center for its ability to help them increase their confidence and comfort with mathematics.

It is critical to consider the physical environment of a support center because it contributes to the improvement or hindrance of students' learning. There are many factors that may go unnoticed such as the temperature, lighting, and noise level until there is a problem [9]. Through photographic studies, surveys, and focus groups, Hunley and Schaller [7] found that students were more engaged in learning spaces that were "comfortable, open, flexible, and appealing to the emotions." Engagement also occurred when the settings encouraged interactions with others, had appropriate technology, comfortable furniture, adequate air circulation and temperature, good lighting, and limited distractions.

To ensure all aspects of the center will fit in the allotted space, it is suggested that the design of the center be created on paper using a scale model. In 2003, a renovation was done on the building that housed the Mathematics Department and the Math Center at Keene State College in Keene, New Hampshire. Figure 1 shows the scale model that was created to show what elements were needed in the new space and how they would work together.

A more recent renovation of a QMaSC space can be found at the Quantitative and Symbolic Reasoning Center at Hamilton College in Clinton, New York [10. Refer to the case studies section at the end of this handbook, where O'Neill writes about the development of the new center space.

Another idea to consider while planning a QMaSC is the use of satellite centers, where tutors provide help for students outside the four walls of the center. Tutors can be given a mobile cart that contains all the necessary items they will need while tutoring. They can hold drop-in times at the library on the weekends or in the residence halls during the late evening when the center is closed. These satellite centers are also helpful for centers that are newly formed and do not have a designated space yet or while a center is under renovation. 


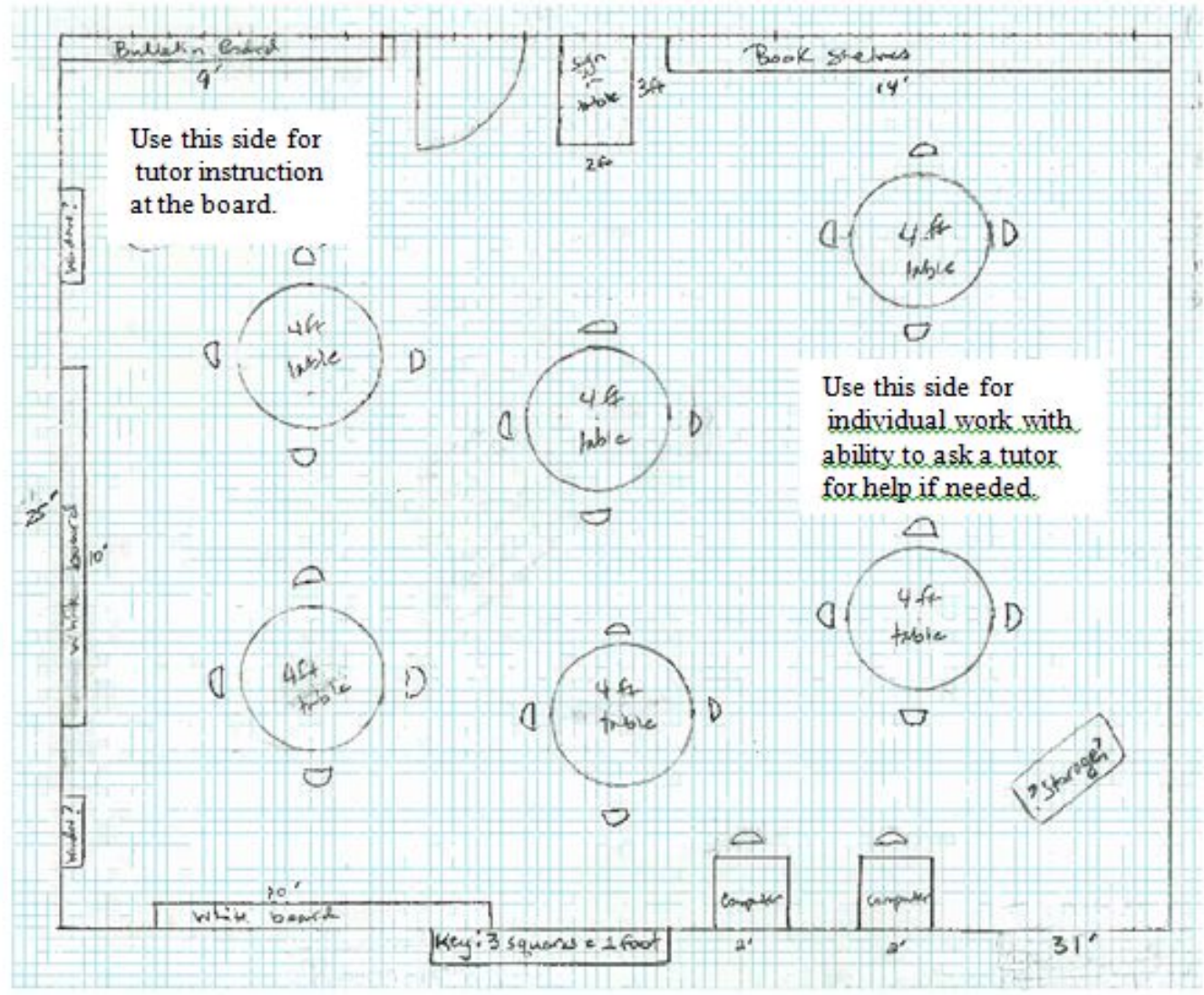

Figure 1: Scale Model for the Math Center at Keene State College

Creating the physical space that will be used on a day-to-day basis requires detailed thought. The remainder of this chapter identifies several components for directors to think about while designing a functional space: tables and chairs, board space, displays, storage, supplies, computers, a reading area, additional rooms, and finishing touches. Each component is explained below.

Tables and Chairs: Many types of tables and chairs can be selected for use in tutoring centers. The director needs to decide what type of furniture will work best for the purposes of the center. Some directors prefer circle tables because they give the students more space to put their textbooks, notes, and other personal items while they work on homework or a project. Circle tables also allow several students to sit comfortably around the table for discussions or group work. Other directors prefer square or rectangular tables so that students can move them around and make different sized tables to fit their needs. Types of chairs also vary from center to center. Some directors prefer rolling chairs for easy movement, whereas others prefer non-fabric chairs for ease of cleaning. As furniture can be very expensive, directors also need to consider the cost of the tables and chairs when making selections. Furniture also needs to be accessible and comfortable to all students, especially larger students or handicapped students [9]. Hunley and Schaller suggest furniture that 
is flexible, "lightweight and easily movable, with a ratio between furniture and free space that allows for alteration of the room setup" [7].

Board Space: Some sort of writing surfaces should be installed to create areas where students can work together on solving problems. Once again, many options are available, including whiteboards, chalkboards, whiteboard or chalkboard paint that goes directly on the walls, glass whiteboards, or even easels. Preference really depends on the individuals using the space. If classes or study sessions will be offered in the center, the needs of those teaching in the space should be taken into consideration as well. For example, will instructors need a projector? If so, then perhaps the writing surface chosen can serve as a screen for the projector.

Displays: Bulletin boards should be placed in the center to display such things as times of operation, announcements to the students and tutors, tutor photos and names, rules of the center, funny math cartoons, and anything else that needs to be visible to the users of the center. Try to keep this area current and changing to hold students' interest.

Storage: Storage space should be incorporated into any center. As they say, one can never have too much storage! Many types of storage should be considered: filing cabinets, bookshelves, display cases, mailboxes, vertical hanging storage, and literature organizers. These organizing units can house such items as past and current textbooks, manipulatives, schedules, handouts on mathematical topics, answer keys, journals, signs for each tutor to put out while on duty, surveys for completion after tutoring, and anything else the tutors may need to perform the duties of their job. Figure 2 shows a photograph of a corner of the Math Center at Keene State College where most of the storage is housed for convenient access. The textbooks shown in the photo are out in the open for students and tutors to use for extra problems or research. Current textbooks are housed in a drawer in a filing cabinet so that they are more secure. Another model to ensure that current textbooks are not taken is described by Croft [3] that textbooks can be "physically secured to the walls or desks." Directors need to provide locked storage for items that are only for the tutors to use and that they do not want to leave the center. Storage should also be provided for the tutors' belongings while they are on duty.

Supplies: Create a work area that contains all the supplies students may need to use at the center (see Figure 2). These items would include a pencil sharpener, stapler, 3-hole punch, tape, pencils/pens, scrap paper, rulers, spare calculators, protractors, compasses, and anything else they may need to execute an assignment.

Computers: Although many centers are equipped with wireless internet, and students will bring 


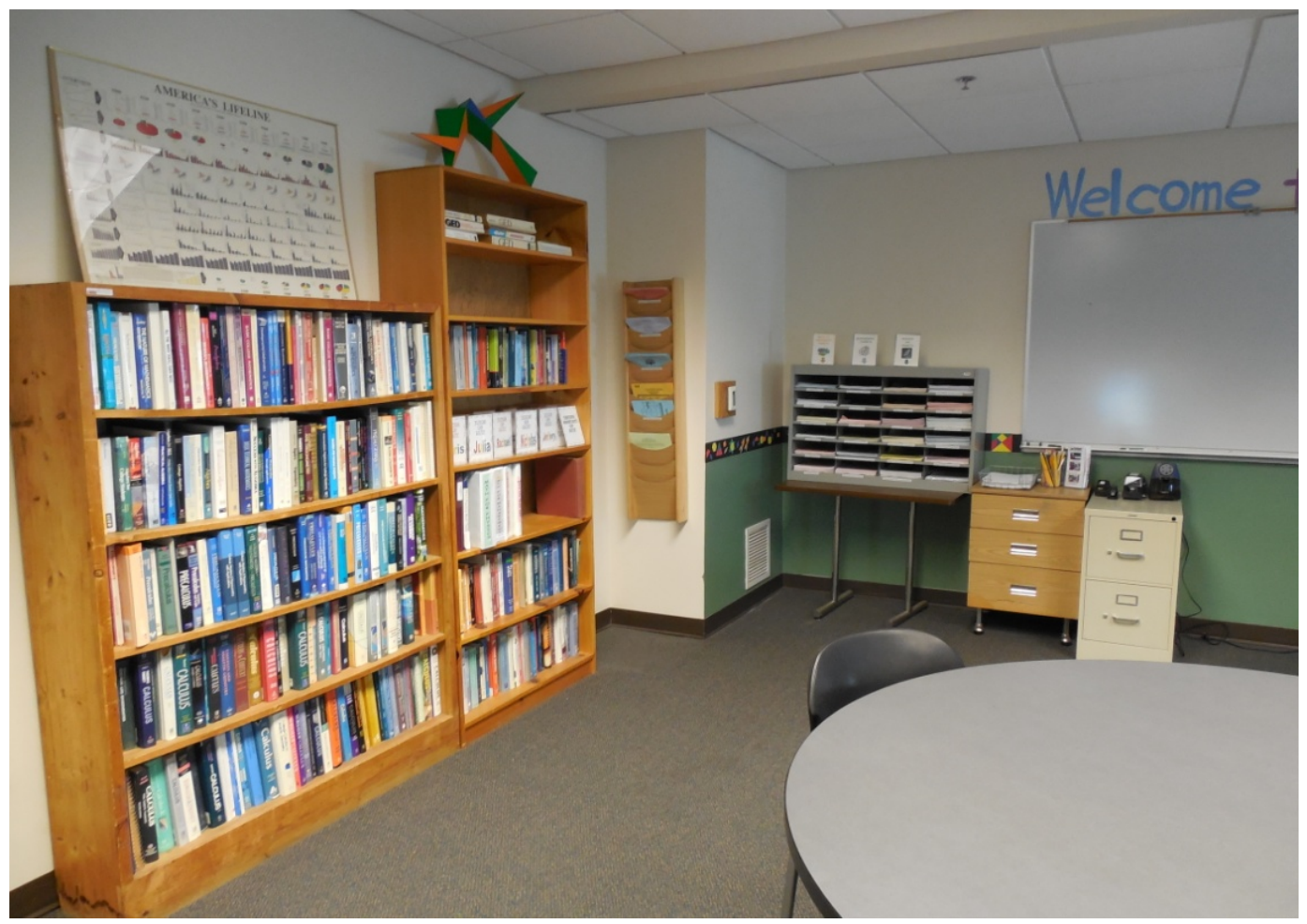

Figure 2: Example Work Area

their own laptops or tablets, it is still important to have at least a couple of desk top computers for students to use at the center. Tutors may need to use the computers to help students with technology-based assignments using mathematical software often not economical for students to have on their personal laptops, or tutors may want to use the computers for their own assignments if there is no one at the center being tutored. Students may also use the computers to complete projects assigned in math or quantitative courses, knowing that someone is there to help them should they run into a problem. Some centers have a computer located near the entrance to use for tracking attendance and survey purposes. For example, according to Cooper [11, the Tutoring Center at Western Washington University used TutorTrac software to electronically track the number of students who visit the center, how many hours they spend at the center, and for what courses they received tutoring help. Other centers use laptops or tablets to record this information. Whatever type of computers the center uses, it is important to keep them secure.

Reading area: If the center has an unused corner, try to create a reading area that is more relaxed with comfortable chairs or a sofa that students can lounge in and read an assignment, catch up on email, or chat with a friend.

Additional rooms: Many centers are housed within the walls of one room. If a center has the luxury 
to have more than one room, the rooms can be assigned for different purposes. Some ideas might be: a quiet area for studying, a testing room, a room for small groups to meet, or a room for majors to hang out.

Finishing touches: Finally, after all the research has been done about the space needs of the center and what items to include in the center, the finishing touches need to be done to make the center an inviting and welcoming place. Here are some ideas: paint the center a calming color, put up colorful and informative posters, paint a border with magnetic and/or chalkboard paint so that colorful magnets can be placed around the room (see Figure 2) or inspirational sayings may be written, have colorful signs throughout the room stating what tutors can and cannot do, post mathematical cartoons, paint the value of $\pi$ on the wall, or have manipulatives displayed in glass display cases to add some color.

\section{Conclusion}

Creating a QMaSC space can be an exciting and challenging endeavor with many factors to consider, from the planning phase to the day-to-day functioning phase. A director should take the time to visit other QMaSC spaces or inquire about the ways in which other QMaSC spaces are designed to find the best practices and use that information to create a center that fits the culture and needs of the individual institution. Center directors should create a welcoming and inviting space that is visible on campus and encourages students to seek the help they need. QMaSC spaces should be flexible so the physical space can be ever-changing with the programmatic needs of the center.

\section{Bibliography}

[1] D. Lawson, Y. Solomon, and A. C. Croft, "Safety in numbers: Mathematics support centres and their derivatives as social learning spaces," Studies in Higher Education, vol. 35, no. 4, pp. 421-431, 2010.

[2] P. Grummon, "Best practices in learning space design: Engaging users," EDUCAUSE Quarterly Magazine, vol. 32, no. 1, 2009. Accessed from http://www.educause.edu/ero/article/ best-practices-learning-space-design-engaging-users June 13, 2013.

[3] A. C. Croft, "A guide to the establishment of a successful mathematics learning support centre," International Journal of Mathematical Education in Science and Technology, vol. 31, no. 3, pp. 431-446, 2000. 
[4] S. Burgstahler, "Tutoring and learning centers." DO-IT, University of Washington, Access College: Student Services Conference Room. Accessed from http://www.washington.edu/ doit/programs/accesscollege/student-services-conference-room/accommodations/ tutoring-and-learning-centers on March 1, 2016.

[5] J. Landsberger, "E-Learning by Design: Academic Support Centers: quo vadis?," TechTrends, vol. 49, no. 4, pp. 8-11, 2005.

[6] C. Halcrow and M. Iiams, "You Can Build It, but Will They Come?," Problems, Resources, and Issues in Mathematics Undergraduate Studies, vol. 21, no. 4, pp. 323-337, 2011.

[7] S. Hunley and M. Schaller, "Assessment the key to creating spaces that promote learning," EDUCAUSE review, vol. 44, no. 2, pp. 26-35, 2009.

[8] C. Carroll and O. Gill, "An innovative approach to evaluating the university of limericks mathematics learning centre," Teaching Mathematics and Its Applications, vol. 31, no. 4, pp. 199-214, 2012.

[9] WGBH Educational Foundation, "Diversity and classroom management: Building a physical space for learning." Retrieved from http://special.league.org/gettingresults/web/ module1/diversity/build_space_learning.html on March 1, 2016, 2006.

[10] M. O'Neill, "A new tutoring center: Challenges, opportunities, and surprises." Retrieved from http://www.hamilton.edu/qsr on August 14, 2013, 2013.

[11] E. Cooper, "Tutoring center effectiveness: The effect of drop-in tutoring," Journal of College Reading and Learning, vol. 40, no. 2, pp. 21-34, 2010. 